\title{
Efeitos Renais e Cardiovasculares da Infusão de Dopamina e da Solução de Cloreto de Sódio a 7,5\%. Estudo Experimental em Cães com Restrição Hídrica *
}

\section{Renal and Cardiovascular Effects of Dopamine and $7.5 \%$ Sodium Chloride Infusion. Experimental Study in Dogs with Water Restriction}

Marisa Aparecida Lima Verderese ${ }^{1}$; Pedro Thadeu Galvão Vianna, TSA ${ }^{2}$; Eliana Marisa Ganem, TSA ${ }^{2}$; Luiz Antonio Vane, TSA ${ }^{2}$

\section{RESUMO}

Verderese MAL, Vianna PTG - Efeitos Renais e Cardiovasculares da Infusão de Dopamina e da Solução de Cloreto de Sódio a 7,5\%. Estudo Experimental em Cães com Restrição Hídrica

JUSTIFICATIVA E OBJETIVOS: É controvertido o uso da infusão de dopamina na proteção renal. O objetivo desta pesquisa foi estudar o efeito da dopamina, da solução hipertônica e da associação de ambas em cães com restrição hídrica, simulando o jejum pré-operatório.

MÉTODO: Foram estudados, em 32 cães anestesiados com tiopental sódico e fentanil, os seguintes parâmetros da função renal: fluxo plasmático efetivo renal (depuração de paraaminohipurato de sódio), ritmo de filtração glomerular (depuração de creatinina) e as depurações de sódio, de potássio e osmolar, excreção fracionária de sódio e potássio, excreção de sódio e potássio e a resistência vascular renal. Os parâmetros cardiovasculares foram: pressão arterial média, freqüência cardíaca, pressão da veia cava inferior, índice cardíaco, hematócrito e índice de resistência vascular periférica. Os animais foram subdivididos, através de sorteio, em 4 grupos experimentais: Grupo 1 - G1 $(n=8)$ - grupo controle; Grupo 2 - G2 $(n=8)$ infusão de dopamina $\left(2 \mu \mathrm{g} \cdot \mathrm{kg}^{-1} \cdot \mathrm{min}^{-1}\right)$, Grupo 3 - $\mathrm{G} 3(n=8)$ solução de cloreto de sódio a 7,5\% $\left(2 \mathrm{ml}^{-1} \mathrm{~kg}^{-1}\right)$ e Grupo 4 - G4 (n= 8) - associação de dopamina ( $\left.2 \mu \mathrm{g} \cdot \mathrm{kg}^{-1} \cdot \mathrm{min}^{-1}\right)$ e cloreto de sódio a $7,5 \%\left(2 \mathrm{ml} . \mathrm{kg}^{-1}\right)$. Os grupos tiveram quatro fases experimentais e cada momento com duração de 30 minutos, compreendendo os momentos M1, M2, M3 e M4.

RESULTADOS: O grupo da dopamina (G2) apresentou diminuição da pressão arterial média, da resistência vascular renal e da excreção de potássio. O grupo da solução hipertônica de cloreto de sódio (G3) apresentou aumento do índice cardíaco, do volume urinário, da depuração de sódio e de potássio, da excreção urinária de sódio e potássio e da excreção fracionária de sódio. No grupo da solução hipertônica de cloreto de sódio associada à dopamina (G4), ocorreu elevação da freqüência cardíaca, do índice cardíaco, do fluxo plasmático efetivo renal e da excreção urinária de sódio; ocorreu também diminuição do índice de resistência vascular sistêmica e do potássio plasmático.

\footnotetext{
* Recebido do (Received from) CET/SBA do Departamento de Anestesiologia da Faculdade de Medicina de Botucatu (FMB - UNESP), Botucatu, SP

1. Pós-Graduanda do Programa de Pós-Graduação em Anestesiologia da FMB - UNESP, em nível de Doutorado

2. Professor (a) Titular do CET/SBA do Departamento de Anestesiologia da FMB - UNESP

Apresentado (Submitted) em 24 de outubro de 2002 Aceito (Accepted) para publicação em 08 de janeiro de 2003

Endereço para correspondência (Correspondence to)

Dr. Pedro Thadeu Galvão Vianna

Dept ${ }^{\circ}$ de Anestesiologia da FMB - UNESP

18618-970 Botucatu, SP

E-mail: ptgv@uol.com.br
}

(C) Sociedade Brasileira de Anestesiologia, 2003
CONCLUSÕES: Deste estudo conclui-se que a solução hipertônica de cloreto de sódio foi capaz de melhorar as condições hemodinâmicas e, conseqüentemente, a função renal de cães sob restrição hídrica de 12 horas. O mesmo não aconteceu com a infusão de $2 \mu \mathrm{g} \cdot \mathrm{kg}^{-1} \cdot \mathrm{min}^{-1}$ de dopamina que, em situação similar, não causou aumento da diurese e da excreção de sódio.

Unitermos: ANIMAL, Cão; DROGAS, Adrenérgicas: dopamina; SISTEMA RENAL: função: VOLEMIA, Expansão: cloreto de sódio a $7,5 \%$

\section{SUMMARY}

Verderese MAL, Vianna PTF - Renal and Cardiovascular Effects of Dopamine and 7.5\% Sodium Chloride Infusion. Experimental Study in Dogs with Water Restriction

BACKGROUND AND OBJECTIVES: Dopamine infusion for renal protection is controversial. This study aimed at observing the effects of dopamine, hypertonic solution and the association of both in dogs with water restriction, emulating preoperative fast. METHODS: The following renal function parameters were studied in 32 dogs anesthetized with sodium pentobarbital and fentanyl: effective renal plasma flow (sodium paraaminohippurate clearance), glomerular filtration rate (creatinine clearance), sodium, potassium and osmolar clearance, sodium and potassium fractional excretion and renal vascular resistance. Cardiovascular parameters were: mean blood pressure, heart rate, inferior vena cava pressure, cardiac index, hematocrit and peripheral vascular resistance index. Animals were randomly distributed in four experimental groups: Group 1 - G1 ( $n=8)$ - control group; Group 2 - G2 ( $n=8)$ - dopamine infusion (2 $\left.\mu \mathrm{g} \cdot \mathrm{kg}^{-1} \cdot \mathrm{min}^{-1}\right)$; Group $3-\mathrm{G} 3(n=8)-7.5 \%$ sodium chloride $\left(2 \mathrm{ml} . \mathrm{kg}^{-1}\right)$ and Group 4 - $\mathrm{G} 4(n=8)$ - association of dopamine $\left(2 \mu \mathrm{g} \cdot \mathrm{kg}^{-1} \cdot \mathrm{min}^{-1}\right)$ and $7.5 \%$ sodium chloride (2 $\left.\mathrm{ml} . \mathrm{kg}^{-1}\right)$. Groups underwent four experimental stages lasting 30 minutes each, and involving moments M1, M2, M3 and M4.

RESULTS: Dopamine group (G2) had mean blood pressure, renal vascular resistance and potassium excretion decrease. Hypertonic sodium chloride group (G3) had cardiac index, urinary volume, sodium and potassium clearance, sodium and potassium urinary excretion and sodium fractional excretion increase. Group receiving the association of hypertonic solution and dopamine (G4) had heart rate, cardiac index, effective renal plasma flow and sodium urinary excretion increase; there has also been systemic vascular resistance and plasma potassium index decrease.

CONCLUSIONS: Our conclusion was that hypertonic sodium chloride solution was able to improve hemodynamic conditions and, as a consequence, renal function of dogs under 12-hour water restriction. The same was not true for $2 \mu \mathrm{g} \cdot \mathrm{kg}^{-1} \cdot \mathrm{min}^{-1} \mathrm{do}-$ pamine which, in a similar situation, has not increased diuresis and sodium excretion.

Key Words: ANIMAL, Dog; DRUGS, Adrenergic: dopamine; RENAL SYSTEM: function; VOLUME STATUS: expansion, $7.5 \%$ sodium chloride 


\section{INTRODUÇÃO}

A infusão de $2 \mu \mathrm{g} \cdot \mathrm{kg}^{-1} \cdot \mathrm{min}^{-1}$ de dopamina aumenta a diurese, a natriurese e o ritmo de filtração glomerular ${ }^{1}$. Contudo, não está estabelecida a existência de efeito protetor renal da dopamina, principalmente quando o rim sofre agressão devido à isquemia ou à hipovolemia. Desse modo, há controvérsias na literatura sobre esse assunto. Assim, há estudos mostrando evidência de melhora da função renal ${ }^{2-4}$ com o uso da dopamina. Outros estudos não obtiveram efeitos diuréticos e natriuréticos com o emprego da infusão da dopamina ${ }^{5,6}$.

O cristalóide hiperosmótico é usado no tratamento do choque hemorrágico ${ }^{7-9}$. A grande vantagem da solução hipertônica de cloreto de sódio a $0,75 \%$ (SH) é a capacidade de reanimar o paciente com volume menor do que o necessário quando se usam soluções isotônicas ${ }^{10}$. Esta vantagem é particularmente útil nos pacientes que necessitam de cuidados pré-hospitalares, principalmente quando não há tempoe nem acesso intravenoso suficiente para infusão de grandes volumes de líquidos. Por esta razão, essa técnica tem ganho enorme aceitação na reanimação e nos primeiros socorros dos pacientes.

O objetivo desta pesquisa foi estudar as ações renais e cardiovasculares da dopamina, da solução hipertônica de cloreto de sódio a $0,75 \%$ e da associação de ambas em cães com restrição hídrica de 12 horas (simulação do jejum pré-anestésico).

\section{MÉTODO}

Após aprovação pela Comissão de Ética em Experimentação Animal Faculdade de Medicina de Botucatu, UNESP, empregaram-se 32 cães adultos, de ambos os sexos, sem raça definida, com peso corpóreo variando de 7 a $17 \mathrm{~kg}$, fornecidos pelo Biotério Central do "Campus" de Botucatu da Universidade Estadual Paulista. Para indução da anestesia, os cães receberam tiopental sódico $15 \mathrm{mg} \cdot \mathrm{kg}^{-1}$, fentanil 15 $\mu \mathrm{g} . \mathrm{kg}^{-1}$ e $60 \mu \mathrm{g} . \mathrm{kg}^{-1}$ de cloreto de alcurônio que foram seguidos pela infusão contínua de $40 \mu \mathrm{g} \cdot \mathrm{kg}^{-1} \cdot \mathrm{min}^{-1}$ de tiopental e $0,1 \mu \mathrm{g} \cdot \mathrm{kg}^{-1} \cdot \mathrm{min}^{-1}$ de fentanil durante todo o período experimental. Os animais foram divididos em quatro grupos experimentais, obedecendo ao critério de sorteio, com oito cães em cada grupo. Grupo 1 - G1 - grupo controle, Grupo 2 - G2 infusão de dopamina $\left(2 \mu \mathrm{g} \cdot \mathrm{kg}^{-1} \cdot \mathrm{min}^{-1}\right)$, Grupo 3 - G3 solução de cloreto de sódio a 7,5\% (SH) (2 ml. $\left.\mathrm{kg}^{-1}\right)$ e Grupo 4 - G4 - associação de dopamina $\left(2 \mu \mathrm{g} \cdot \mathrm{kg}^{-1} \cdot \mathrm{min}^{-1}\right)$ e do cloreto de sódio a $7,5 \%\left(2 \mathrm{ml}^{\mathrm{kg}} \mathrm{kg}^{-1}\right)$. Nos grupos G2 e G4 foi iniciada a infusão de dopamina no final do momento 1 (M1); neste mesmo período, os animais dos grupos G3 e G4 receberam a injeção venosa de $\mathrm{SH}$.

Os atributos foram estudados nos seguintes momentos: momento 1 (M1): obtido 30 minutos após a infusão das doses iniciais de PAH (para-aminohipurato de sódio) e de creatinina; Momentos 2 (M2), 3 (M3) e 4 (M4) obtidos, respectivamente, 30,60 e 90 minutos após o início do tratamento correspon- dente ao grupo sorteado. Os momentos tiveram duração de 30 minutos.

Em todos os animais foi realizada a seguinte seqüência experimental: jejum alimentar e restrição hídrica de 12 horas; pesagem do animal; anestesia; colocação e fixação do cão sobre goteira de Claude Bernard; intubação traqueal, instalação da respiração controlada e ventilação dos animais com ar comprimido, empregando-se o aparelho de anestesia K. Takaoka, modelo 850-10. Dissecção dos vasos sangüíneos para hidratação $\left(0,03 \mathrm{ml} \cdot \mathrm{kg}^{-1} \cdot \mathrm{min}^{-1}\right)$, toracotomia no $4^{\circ}$ espaço intercostal para instalação da sonda fluxométrica na porção inicial da aorta ascendente, adaptação e calibração do fluxômetro eletromagnético (Blood Flowmeter) - Gould Statham, modelo SP 2202; injeção da dose inicial de PAH e creatinina (solução de PAH a 0,4\% e creatinina a $3 \%$ no volume de $1 \mathrm{ml} . \mathrm{kg}^{-1}$ ) e a seguir infusão contínua de PAH a 1,6\% e creatinina a $4 \%$ em solução de glicose a $5 \%\left(0,015 \mathrm{ml}^{\mathrm{kg}} \mathrm{kg}^{-1} \cdot \mathrm{min}^{-1}\right)$; todos os animais receberam dose complementar de cloreto de alcurônio $\left(60 \mu \mathrm{g} \cdot \mathrm{kg}^{-1}\right)$. No final do experimento os cães foram sacrificados com injeção de cloreto de potássio.

Foram estudados os seguintes atributos: pressão arterial média (PAM); freqüência cardíaca (FC); pressão da veia cava inferior (PVCI); índice cardíaco $(\mathrm{IC})$; hematócrito $(\mathrm{Ht})$; índice de resistência vascular periférica (IRVP); medidas da depuração de creatinina- ritmo de filtração glomerular (RFG) e do para-aminohipurato de sódio-fluxo plasmático efetivo renal (FPER); resistência vascular renal (RVR) sódio, potássio e osmolalidade plasmática e urinária; temperatura retal $\left({ }^{\circ} \mathrm{C}\right)$.

Para cada variável foram calculadas a média $(x)$ e o desvio padrão (s) em cada um dos momentos e foi utilizada a análise de variância, fatorial inteiramente aleatória com testes de: interação entre grupo e momento, efeito de grupo e efeito de momento. Em todas as hipóteses testadas, as estatísticas $\mathrm{F}$ calculadas foram consideradas significativas quando $p<$ 0,05 . Os contrastes entre pares de médias foram analisados pelo teste de Tukey, com cálculo da diferença mínima significativa para alfa $=0,05$.

\section{RESULTADOS}

Os grupos foram homogêneos com relação à idade, ao peso e ao sexo.

Em todos os quatro grupos houve valores elevados da osmolalidade urinária que diminuíram no decorrer do experimento (Tabela I e Figura 1, F = 29,1 p<0,05 M1 > (M3 = M4) M2, intermediário).

Ainfusão de dopamina $\left(2 \mu \mathrm{g} \cdot \mathrm{kg}^{-1} \cdot \mathrm{min}^{-1}\right)$ aumentou o hematócrito $(F=7,85 ; p<0,05)$, diminuiu a PAM $(F=5,96 ; p<0,05)$ e a $\operatorname{RVR}(F=6,65 ; p<0,05)$.

Ainfusão de SH proporcionou o aumento de: 1. Índice cardíaco (Tabela l e Figura 2) efeitos de momentos: $F=5,45 p<0,05$ $(\mathrm{M} 1=\mathrm{M} 2=\mathrm{M} 3)>\mathrm{M} 4 ; \mathrm{G} 3: \mathrm{F}=16,57 \mathrm{p}<0,05 \mathrm{M} 2>(\mathrm{M} 3=\mathrm{M} 4)$ $M 1$ intermediário. 2. Hematócrito $(F=14,47 ; p<0,05) .3$. Osmolalidade e sódio plasmáticos $(F=6,74 ; p<0,05$ e $F=$ 7,59; $p<0,05$ ). 4. Volume urinário (Tabela I e Figura 3) efeito 
Tabela I - Resultados da Osmolalidade Urinária, Índice Cardíaco, Volume Urinário, Excreção Urinária de Sódio e Fluxo Plasmático Efetivo Renal

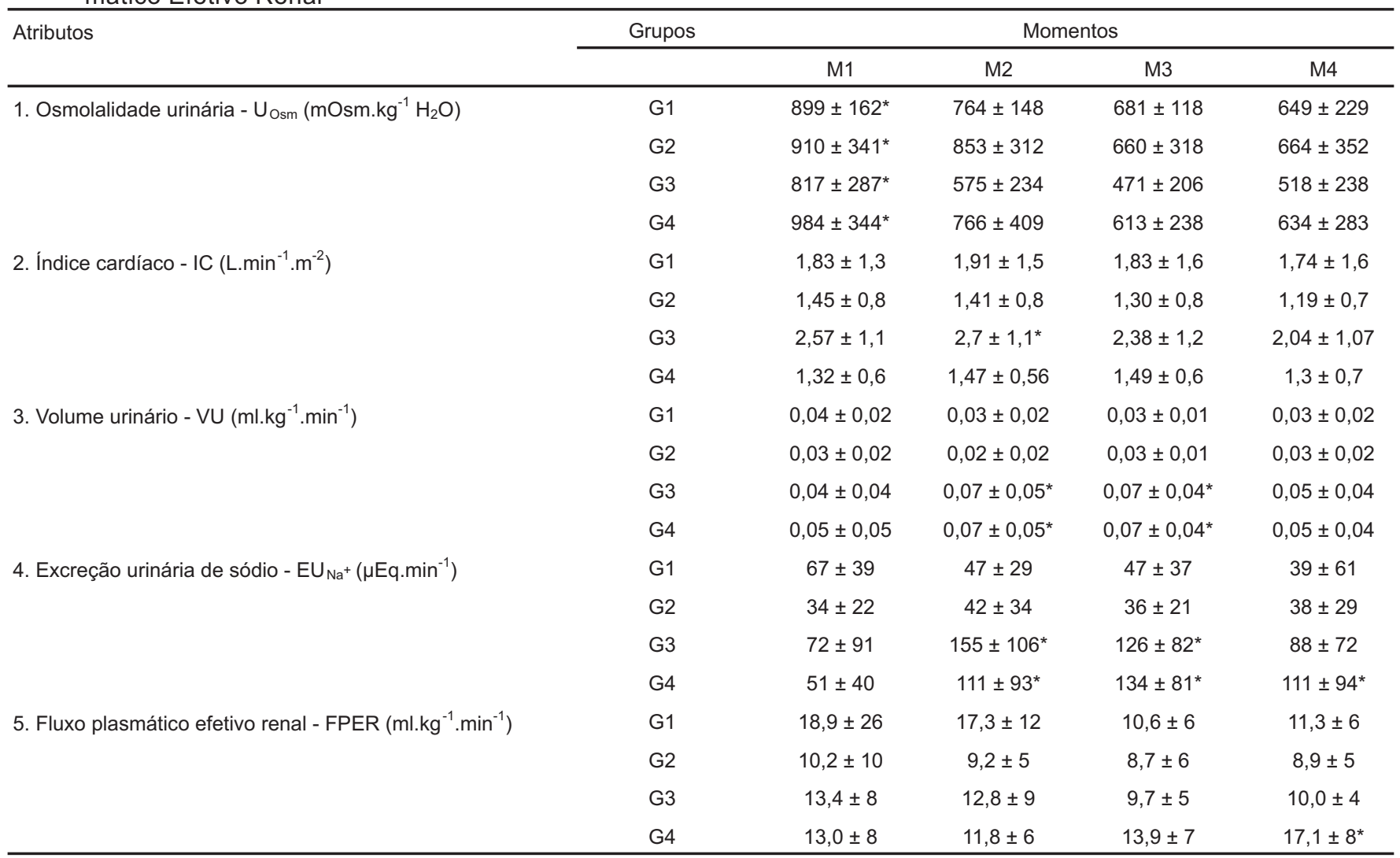

${ }^{*} p<0,05$

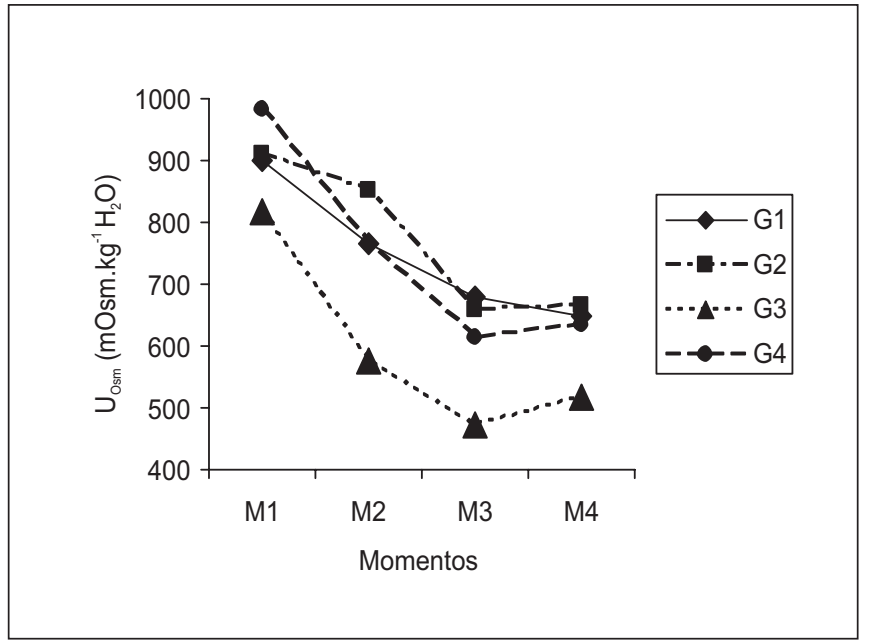

Figura 1 - Osmolalidade Urinária $U_{\mathrm{Osm}}\left(\mathrm{mOsm} . \mathrm{kg}^{-1} \mathrm{H}_{2} \mathrm{O}\right)$ em Relação ao Tempo (minutos)

G1 (grupo controle); G2 (grupo dopamina); G3 (grupo SH); G4 (grupo SH + dopamina)

de grupo $F=3,05 p<0,05(G 1=G 2)<(G 3=G 4)$; efeito de grupo em cada momento $M 2: F=3,51 p<0,05(G 1=G 2)<(G 3=$ G4); $M 3: F=4,3 p<0,05(G 1=G 2)<(G 3=G 4) 5$. Excreção urinária de sódio (Tabela le Figura 4) efeito de grupo em cada momento M2: $F=4,34 p<0,05(\mathrm{G} 1=\mathrm{G} 2)<\mathrm{G} 3$ e G4 intermediário; $M 3$ : $F=5,59 p<0,05(G 1=G 2)<(G 3=G 4)$; efeito de momento em cada grupo G3: $F=14,42 p<0,05(M 1=M 4)<$ M2 e M3 intermediário.

Com a infusão de $\mathrm{SH}$, associada à dopamina, ocorreu elevação de: 1) Freqüência cardíaca $(F=10,52 ; p<0,05) ; 2) F P E R$ (Tabela le Figura 5) efeito de grupo em cada momento M4:F= 3,06 $p<0,05(G 1=G 2=G 3)<G 4$ e efeito de momento em cada grupo $G 4: F=5,64 p<0,05(M 1=M 2=M 3)<M 4 ; 3)$ Sódio plasmático $(F=6,32 ; p<0,05) ; 4)$ Excreção urinária de sódio (Tabela I e Figura.4) efeito de momento em cada grupo $\mathrm{G} 4: \mathrm{F}=7,77 \mathrm{p}<0,05 \mathrm{M} 1<(\mathrm{M} 2=\mathrm{M} 3=\mathrm{M} 4)$; ocorreu também diminuição de: 1) índice de resistência vascular sistêmica ( $F=$ $13,87 ; p<0,05) ; 2)$ potássio plasmático $(F=10,9 p<0,05)$.

\section{DISCUSSÃO}

A técnica de infusão contínua do tiopental sódico associado ao fentanil produziu estabilidade hemodinâmica e renal a estes animais e, conseqüentemente, não houve alteração dos principais parâmetros renais e cardiovasculares. Aosmolalidade urinária que apresentou valores elevados diminuiu do momento M1 para o M4. De modo semelhante a outras pesquisas, estes valores elevados da osmolalidade demonstraram que a restrição hídrica, simulando o jejum 


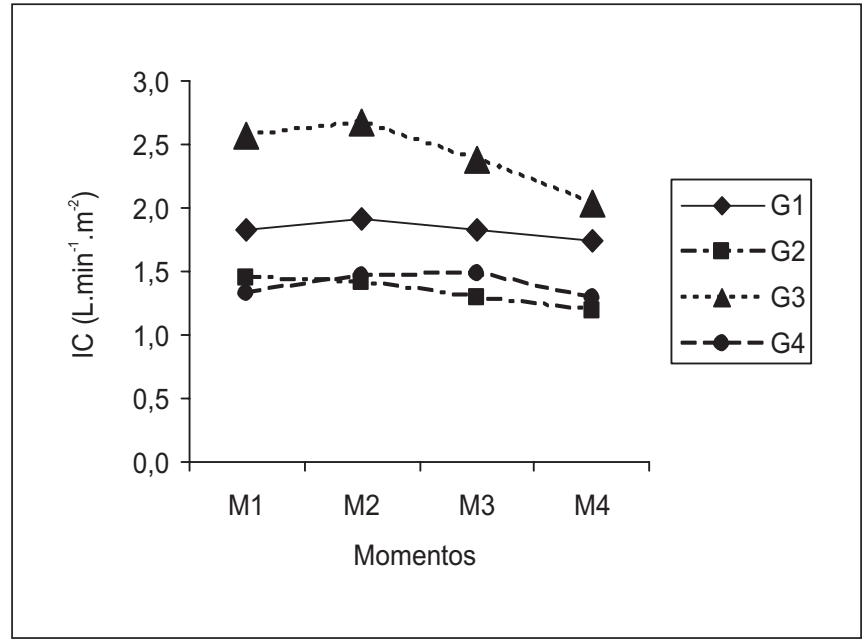

Figura 2 - Índice Cardíaco IC $\left(\right.$ L. $\left.\mathrm{min}^{-1} \cdot \mathrm{m}^{-2}\right)$ em Relação ao Tempo (minutos)

G1 (grupo controle); G2 (grupo dopamina); G3 (grupo SH); G4 (grupo SH + dopamina)

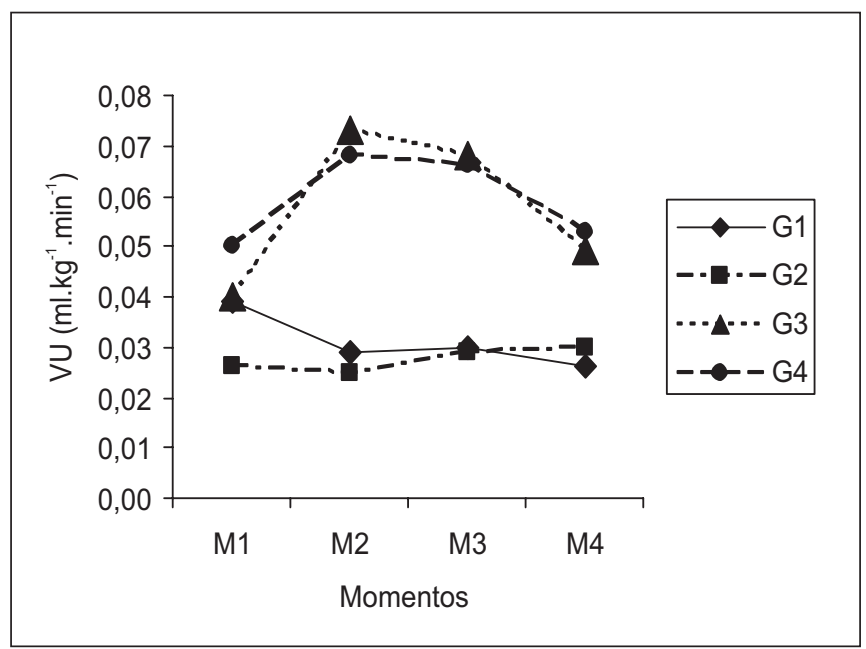

Figura 3 - Volume Urinário - VU ( $\left.\mathrm{ml}^{\mathrm{kg}}{ }^{-1} \cdot \mathrm{min}^{-1}\right)$ em Relação ao Tempo (minutos)

G1 (grupo controle); G2 (grupo dopamina); G3 (grupo SH); G4 (grupo SH + dopamina)

pré-operatório, foi suficiente para causar a contração do volume extracelular.

Sabe-se que a dopamina tem ação dose-dependente, baseada na sua atividade sobre os receptores dopaminérgicos, $\beta$-adrenérgicos ou $\alpha$-adrenérgicos. A infusão de dopamina acima de $2,5 \mu \mathrm{g} \cdot \mathrm{kg}^{-1} \cdot \mathrm{min}^{-1}$ apresenta efeitos cardiovasculares através dos receptores $\beta$-adrenérgicos ${ }^{11,12}$. Geralmente doses menores causam mínimos efeitos sistêmicos, mas alguns estudos sugerem que existe significante variação individual. Nos indivíduos normais, a infusão de $2,5 \mu \mathrm{g} \cdot \mathrm{kg}^{-1} \cdot \mathrm{min}^{-1}$ de dopamina diminuiu a resistência vascular sistêmica em $27 \%^{11}$.

No nosso estudo, os valores de freqüência cardíaca (FC), índice cardíaco (IC), pressão da veia cava inferior (PVCI) e ín-

Revista Brasileira de Anestesiologia

Vol. 53, № 5, Setembro - Outubro, 2003

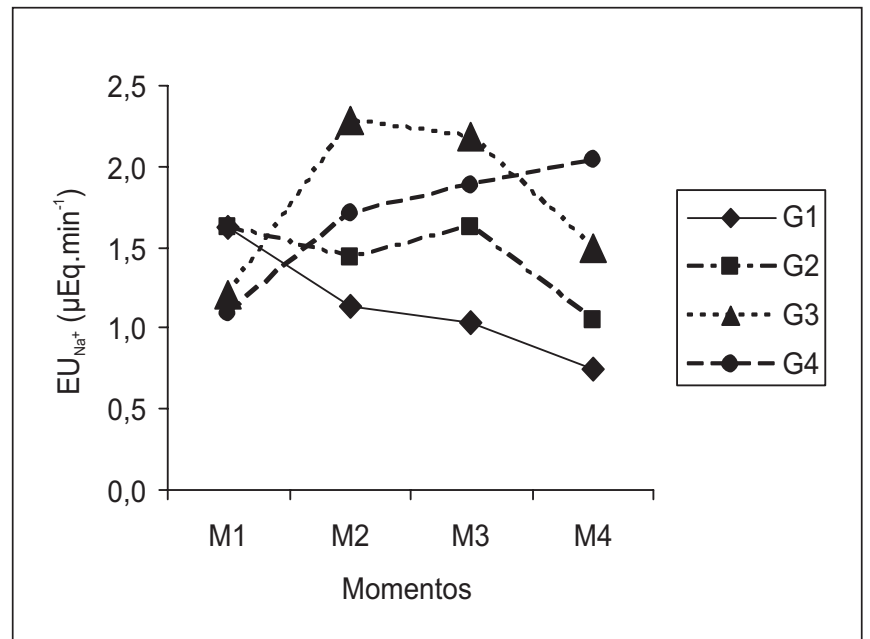

Figura 4 - Excreção Urinária de sódio $\mathrm{EU}_{\mathrm{Na}}{ }^{+}\left(\mu \mathrm{Eq} \cdot \mathrm{min}^{-1}\right)$ em Relação ao Tempo (minutos)

G1 (grupo controle); G2 (grupo dopamina); G3 (grupo SH); G4 (grupo $\mathrm{SH}+$ dopamina)

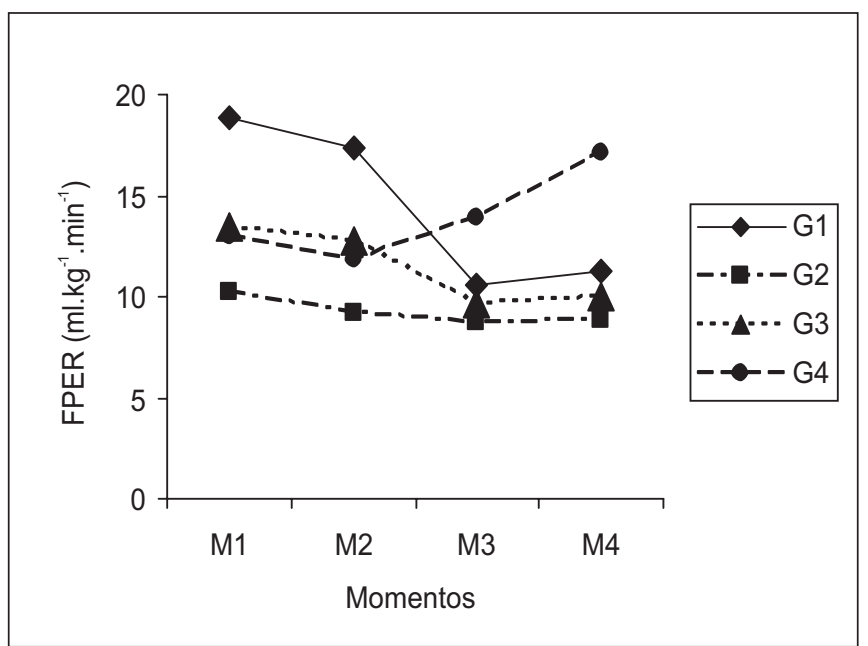

Figura 5 - Fluxo Plasmático Efetivo Renal - FPER $\left(\mathrm{ml}^{\mathrm{kg}} \mathrm{kg}^{-1} \cdot \mathrm{min}^{-1}\right) \mathrm{em}$ Relação ao Tempo (minutos)

G1 (grupo controle); G2 (grupo dopamina); G3 (grupo SH); G4 (grupo $\mathrm{SH}+$ dopamina)

dice de resistência vascular periférica (IRVP) não se alteraram, à semelhança do que ocorre na maioria dos trabalhos que utilizaram a dose de $2 \mu \mathrm{g} \cdot \mathrm{kg}^{-1} \cdot \mathrm{min}^{-1}$ de dopamina. APAM diminuiu após o início da infusão de dopamina, mostrando que, nestas concentrações, essa catecolamina possui efeito vasodilatador. Entretanto, esse efeito não foi intenso, deixando esse atributo dentro dos limites da normalidade. Desde que alguns estudos ${ }^{13-15}$ mostraram que a dopamina aumenta o FSR e o débito urinário, doses baixas de dopamina começaram a ser opção na prevenção de distúrbios renais em pacientes graves. Foi demonstrado aumento do volume urinário na necrose tubular aguda oligúrica, com a infusão de dopamina ${ }^{2}$. O volume urinário e a depuração da creatinina aumentam com a infusão da dopamina, em pacientes sub- 
metidos à transplante de fígado ${ }^{4}$. Estes resultados não foram confirmados posteriormente ${ }^{5}$, quando os autores não encontraram aumento da diurese com a dopamina. Sugere-se ser o grau de hidratação dos pacientes a provável causa dessa discrepância entre os estudos realizados.

Há trabalhos com pacientes em diversas situações (hígidos, gravemente enfermos, com hipertensão intracraniana, no pós-operatório de icterícia obstrutiva e na cirurgia cardíaca), que mostram aumento do volume urinário com a infusão de dopamina ${ }^{1,14,16}$, porém, diferentemente dos animais do presente estudo, estes indivíduos estavam euvolêmicos.

Os resultados desta pesquisa também são concordantes com outro estudo ${ }^{13}$, no qual se utilizou infusão de 0,5 $\mu \mathrm{g} \cdot \mathrm{kg}^{-1} \cdot \mathrm{min}^{-1}$ de dopamina, não tendo sido encontradas alterações no débito urinário, na depuração de água livre e na osmolalidade plasmática, mas mostrando diminuição da resistência vascular renal. Também não se encontrou aumento do volume urinário ao infundir dopamina em recém-nascidos prematuros ${ }^{17}$. Os autores acreditam que este fato pode se dar devido à retração de volume, o que ficou evidenciado pelos elevados valores da osmolaridade urinária. A administração de dopamina em pacientes no pré-operatório de cirurgia cardíaca causou aumento da diurese e natriurese apenas nos pacientes previamente hidratados; enquanto que nos pacientes em jejum e com restrição hídrica não ocorreram estas alterações ${ }^{6}$. Pacientes oligúricos, em cuidados intensivos, não tiveram aumento significativo da excreção urinária e da excreção fracionária de sódio ${ }^{16}$.

Baseado nos resultados desses estudos e da presente pesquisa, pode-se sugerir que, para existir o efeito diurético e o natriurético da dopamina, é necessário que os pacientes tenham um volume extracelular normal ou aumentado.

Nesta pesquisa, a infusão de dopamina elevou o FPER em M4 no G4 (grupo da dopamina associada à SH), mostrando o efeito vasodilatador renal da dopamina que é comprovada pela diminuição da RVR. Entretanto, a dopamina pode, também, produzir concomitante vasodilatação esplâncnica. Este efeito vasodilatador esplâncnico pode diminuir ainda mais a volemia circulante ${ }^{18}$.

Existe pesquisa mostrando elevação do hematócrito com doses altas de dopamina, mas não quando são utilizadas doses pequenas ${ }^{19}$. O aumento do hematócrito em nosso estudo pode ser atribuído à restrição hídrica durante a pesquisa experimental.

A infusão de solução hipertônica de cloreto de sódio a 7,5\% aumenta o débito cardíaco, a PAM e a PVCI, porém causa diminuição da resistência vascular periférica total $7,10,20$. Embora esta diminuição da resistência vascular periférica total resulte parcialmente da expansão do volume intravascular, a ação vasodilatadora direta da hiperosmolalidade pode também contribuir ${ }^{10}$. O efeito vasodilatador direto da hiperosmolalidade pode ajudar a explicar o aumento do índice cardíaco no presente estudo. A solução hipertônica de cloreto de sódio restaura rapidamente o volume intravascular pela captação de água do espaço intracelular para o espaço intersticial e intravascular ${ }^{21}$. Amelhora do débito cardíaco parece ser independente da melhora da pré-carga. $\mathrm{O}$ au- mento do débito cardíaco pode ser por efeito direto sobre a contratilidade ${ }^{22}$ ou por extensa vasodilatação pré-capilar 10,23

Ainfusão de solução hipertônica de cloreto de sódio não alterou a PAM e a PVCI, diferindo de alguns trabalhos que mostraram elevação destes parâmetros ${ }^{7,10}$. Nesta pesquisa, a freqüência cardíaca também não se alterou no grupo G3, e no grupo G4. Possivelmente, a explicação para estes resultados foi a restrição hídrica a que foram submetidos os cães deste estudo e a estabilidade anestésica conseguida pelo uso da infusão contínua do tiopental sódico e fentanil.

Como esperado, o índice cardíaco aumentou no grupo G3, de modo semelhante ao ocorrido em vários trabalhos $8-10,20,24,25$, que mostraram aumento do débito cardíaco com infusão de SH, aumento que foi transitório ${ }^{10,20}$.

Neste estudo, a diminuição do IRVP nos grupos G3 e G4, após a infusão de solução hipertônica de cloreto de sódio, é concordante com a obtida por diversos autores ${ }^{10,20,25}$

Em suínos, a infusão de solução hipertônica de cloreto de sódio aumenta o débito cardíaco e o FSR ${ }^{24}$.

Nosso trabalho teve resultados concordantes com outros $7,10,20,24,26$, que também encontraram aumento do volume urinário, do potássio plasmático, da excreção urinária de sódio e da osmolalidade plasmática, com a infusão de solução hipertônica. ASH promove expansão do volume extracelulare, como já foi dito, isto leva à inibição do sistema RAA (Renina-Angiotensina-Aldosterona), determinando aumento da excreção urinária de sódio. Isto explica o aumento estatisticamente significante da excreção urinária de sódio nos grupos G3 e G4, no nosso experimento. Também explica o aumento da excreção fracionária de sódio nestes grupos, sendo significativo no grupo G3.

A diminuição do potássio plasmático nos grupos em que foi administrada a solução hipertônica de cloreto de sódio foi concordante com vários trabalhos ${ }^{10,20,25}$. Sugere-se que, provavelmente, possa ter ocorrido hipopotassemia pela passagem do $\mathrm{K}^{+}$para o espaço intracelular, embora a resolução tenha sido espontânea.

Concluindo: (a) a dopamina na dose de $2 \mu \mathrm{g} \cdot \mathrm{kg}^{-1} \cdot \mathrm{min}^{-1}$ causou diminuição significativa da pressão arterial média e da resistência vascular renal; (b) a solução hipertônica de cloreto de sódio alterou significativamente a hemodinâmica cardiovascular, com efeito inotrópico, alterou a hemodinâmica renal, com aumento da depuração de sódio e potássio. Causou também alteração na função renal com aumento do volume urinário, da excreção urinária de sódio e potássio e da excreção fracionária de sódio. Diminuiu a osmolalidade urinária. Todas as alterações foram transitórias;(c) a solução hipertônica de cloreto de sódio associada à dopamina alterou significativamente a hemodinâmica cardiovascular, com efeitos cronotrópicos e inotrópicos. Alterou também a hemodinâmica renal, com aumento do fluxo plasmático efetivo renal e da depuração de água livre. Alterou a função renal, com aumento da excreção urinária de sódio e diminuição da osmolalidade. 


\section{Renal and Cardiovascular Effects of Dopamine and $7.5 \%$ Sodium Chloride Infusion. Experimental Study in Dogs with Water Restriction}

Marisa Aparecida Lima Verderese, M.D.; Pedro Thadeu Galvão Vianna, TSA, M.D.; Eliana Marisa Ganem, TSA, M.D.; Luiz Antonio Vane, TSA, M.D.

\section{INTRODUCTION}

The infusion of $2 \mu \mathrm{g} \cdot \mathrm{kg}^{-1} \cdot \mathrm{min}^{-1}$ dopamine increases diuresis, natriuresis and glomerular filtration rate ${ }^{1}$. However, dopamine's renal protective effect is not yet established, especially when kidneys are aggressed by ischemia or hypovolemia. So, literature is controversial about the subject. There are studies showing evidences of improved renal function ${ }^{2-4}$ with dopamine, but other studies have not observed diuretic and natriuretic effects of dopamine ${ }^{5,6}$.

Hyperosmotic crystalloids are used to treat hemorrhagic shock $^{7-9}$. Major $0.75 \%$ hypertonic sodium chloride solution advantage is the ability to reanimate patients with volumes below those needed when isotonic solutions are used ${ }^{10}$. This is particularly useful for patients needing pre-hospital care, especially when there is no time or sufficient venous access for large volume infusions. So, this technique is becoming increasingly popular for patients' resuscitation and first aid. Our study aimed at evaluating renal and cardiovascular effects of dopamine, $0.75 \%$ hypertonic sodium chloride solution and the association of both in dogs with 12-hour water restriction (preanesthetic fast simulation).

\section{METHODS}

After the Animal Experiment Ethics Committee, Faculdade de Medicina, Botucatu, UNESP, approval 32 adult mixed-breed dogs of both genders, weighing 7 to $17 \mathrm{~kg}$, supplied by the experimental animals facility of the Botucatu campus, Universidade Estadual Paulista, were involved in the experiment. Anesthesia was induced with $15 \mathrm{mg}^{\mathrm{kg}}{ }^{-1} \mathrm{so}-$ dium thiopental, $15 \mu \mathrm{g} \cdot \mathrm{kg}^{-1}$ fentanyl and $60 \mu \mathrm{g} \cdot \mathrm{kg}^{-1}$ alcuronium, followed by $40 \mu \mathrm{g} \cdot \mathrm{kg}^{-1} \cdot \mathrm{min}^{-1}$ thiopental and 0.1 $\mu \mathrm{g} \cdot \mathrm{kg}^{-1} \cdot \mathrm{min}^{-1}$ fentanyl continuous infusion throughout the experiment. Animals were randomly distributed in four experimental groups of 8 dogs. Group 1 - G1 - control group; Group 2 - G2 - dopamine infusion (2 $\left.\mu \mathrm{g} \cdot \mathrm{kg}^{-1} \cdot \mathrm{min}^{-1}\right)$; Group 3-G3-7.5\% sodium chloride solution (SH) (2 ml. $\left.\mathrm{kg}^{-1}\right)$; and Group 4 - G4 association of dopamine $\left(2 \mu \mathrm{g} \cdot \mathrm{kg}^{-1} \cdot \mathrm{min}^{-1}\right)$ and $7.5 \%$ sodium chloride $\left(2 \mathrm{ml}^{\mathrm{kg}} \mathrm{kg}^{-1}\right)$. Dopamine infusion was started in groups G2 and G4 at the end of moment M1; at the same time, G3 and $\mathrm{G} 4$ animals received intravenous $\mathrm{SH}$ injections.

Attributes were studied in the following moments: M1 - 30 minutes after initial PAH (sodium para-aminohippurate) and creatinine doses; M2, M3 and M4 30, 60 and 90 minutes after

Revista Brasileira de Anestesiologia

Vol. 53, No 5, Setembro - Outubro, 2003 beginning of the treatment corresponding to the group, respectively. Moments lasted 30 minutes.

The following experimental sequence was adopted for all animals: 12-hour fast and water restriction; animals' weighing; animals' placement and fixation on Claude-Bernard device; tracheal intubation; controlled breathing and ventilation with compressed air using the K. Takaoka, model 850-10 anesthesia machine; blood vessels dissection for hydration $(0.03$ $\left.\mathrm{ml} . \mathrm{kg}^{-1} \cdot \mathrm{min}^{-1}\right)$; thoracotomy at the $4^{\text {th }}$ intercostal space for flowmeter probe insertion at the initial portion of the ascending aorta; adjustment and gaging of electromagnetic blood flowmeter - Gould Statham, model SP 2202; first PAH and creatinine injection $(0.4 \% \mathrm{PAH}$ solution and $3 \%$ creatinine in a volume of $1 \mathrm{ml}^{\mathrm{kg}} \mathrm{kg}^{-1}$ body weight) followed by $1.6 \% \mathrm{PAH}$ and $4 \%$ creatinine in $5 \%$ glucose continuous infusion $(0.015$ $\left.\mathrm{ml} . \mathrm{kg}^{-1} \cdot \mathrm{min}^{-1}\right)$; all animals received additional alcuronium dose $\left(60 \mu \mathrm{g} \cdot \mathrm{kg}^{-1}\right)$. Animals were sacrificed at the end of the experiment with potassium chloride.

The following attributes were studied: mean blood pressure (MBP); heart rate (HR); inferior vena cava pressure (IVCP); cardiac index $(\mathrm{Cl})$; hematocrit $(\mathrm{Ht})$; peripheral vascular resistance index (PVRI); creatinine - glomerular filtration rate (GFR) clearance and sodium para-aminohippurate - effective renal plasma flow (ERPF) clearance; renal vascular resistance (RVR), sodium, potassium and plasma and urinary osmolality; rectal temperature $\left({ }^{\circ} \mathrm{C}\right)$. The following were calculated for each variable: mean (x) and standard deviation (s) in each moment, and totally factorial randomized analysis of variance with interaction tests among groups and moments, group effect and moment effect. In all tested hypothesis, F statistics calculated were considered significant when $p<$ 0.05 . Contrasts among mean pairs were analyzed by Tukey's test with minimum significant difference calculation for $\alpha=$ 0.05 .

\section{RESULTS}

Groups were homogeneous in age, weight and gender. There were high urinary osmolality values in all groups, which have decreased along the experiment (Table I and Figure 1, $F$ $=29.1 \mathrm{p}<0.05 \mathrm{M} 1>(\mathrm{M} 3=\mathrm{M} 4) \mathrm{M} 2$, intermediate $)$.

Dopamine infusion $\left(2 \mu \mathrm{g} \cdot \mathrm{kg}^{-1} \cdot \mathrm{min}^{-1}\right)$ has increased hematocrit $(F=7.85 ; p<0.05)$ and decreased $M B P(F=5.96$; $p>0.05)$ and $R V R(F=6.65 ; p<0.05)$.

SH infusion has increased: 1 ) cardiac index (Table I and Figure 2) moment effects: $F=5.45 p<0.05(M 1=M 2=M 3)>M 4$; G3: $F=16.57 p<0.05 M 2>(M 3=M 4) M 1$ intermediate. 2$) \mathrm{He}-$ matocrit $(F=14.47 p<0.05)$.3) Osmolality and plasma sodium $(F=6.74 p<0.05$ and $F=7.59 p<0.05) .4)$ Urinary volume (Table l and Figure 3) group effect $F=3.05 p<0.05$ (G1 $=\mathrm{G} 2<$ (G3 = G4) group effect in each moment M2: $F=3.51 p<0.05$ $(\mathrm{G} 1=\mathrm{G} 2)<(\mathrm{G} 3=\mathrm{G} 4)$; M3: $\mathrm{F}=4.3 \mathrm{p}<0.05(\mathrm{G} 1=\mathrm{G} 2)<(\mathrm{G} 3=$ G4). 5) Sodium urinary excretion (Table I and Figure 4) group effect in each moment M2: $F=4.34 p<0.05(G 1=G 2)<G 3$ and G4 intermediate; M3: F $=5.59 p<0.05(\mathrm{G} 1=\mathrm{G} 2)<(\mathrm{G} 3=$ G4); moment effect in each group: $F=14.42 p<0.05$ (M1 = M4) < M2 and M3 intermediate. 
Table I - Urinary Osmolality, Cardiac Index, Urinary Volume, Sodium Urinary Excretion and Effective Renal Plasma Flow

\begin{tabular}{|c|c|c|c|c|c|}
\hline \multirow[t]{2}{*}{ Attributes } & \multirow[t]{2}{*}{ Groups } & \multicolumn{4}{|c|}{ Moments } \\
\hline & & M1 & M2 & M3 & M4 \\
\hline \multirow[t]{4}{*}{ 1. Urinary osmolality - UOsm (mOsm. $\left.\mathrm{kg}^{-1} \mathrm{H}_{2} \mathrm{O}\right)$} & G1 & $899 \pm 162^{*}$ & $764 \pm 148$ & $681 \pm 118$ & $649 \pm 229$ \\
\hline & $\mathrm{G} 2$ & $910 \pm 341^{*}$ & $853 \pm 312$ & $660 \pm 318$ & $664 \pm 352$ \\
\hline & G3 & $817 \pm 287^{*}$ & $575 \pm 234$ & $471 \pm 206$ & $518 \pm 238$ \\
\hline & G4 & $984 \pm 344^{*}$ & $766 \pm 409$ & $613 \pm 238$ & $634 \pm 283$ \\
\hline \multirow[t]{4}{*}{ 2. Cardiac index $-\mathrm{Cl}\left(\mathrm{L} \cdot \mathrm{min}^{-1} \cdot \mathrm{m}^{-2}\right)$} & G1 & $1.83 \pm 1.3$ & $1.91 \pm 1.5$ & $1.83 \pm 1.6$ & $1.74 \pm 1.6$ \\
\hline & G2 & $1.45 \pm 0.8$ & $1.41 \pm 0.8$ & $1.30 \pm 0.8$ & $1.19 \pm 0.7$ \\
\hline & G3 & $2.57 \pm 1.1$ & $2.7 \pm 1.1^{*}$ & $2.38 \pm 1.2$ & $2.04 \pm 1.07$ \\
\hline & G4 & $1.32 \pm 0.6$ & $1.47 \pm 0.56$ & $1.49 \pm 0.6$ & $1.3 \pm 0.7$ \\
\hline \multicolumn{6}{|l|}{ 3. Urinary volume - UV $\left(\mathrm{ml} \cdot \mathrm{kg}^{-1} \cdot \mathrm{min}^{-1}\right)$} \\
\hline & G1 & $0.04 \pm 0.02$ & $0.03 \pm 0.02$ & $0.03 \pm 0.01$ & $0.03 \pm 0.02$ \\
\hline & G2 & $0.03 \pm 0.02$ & $0.02 \pm 0.02$ & $0.03 \pm 0.01$ & $0.03 \pm 0.02$ \\
\hline & G3 & $0.04 \pm 0.04$ & $0.07 \pm 0.05^{*}$ & $0.07 \pm 0.04^{*}$ & $0.05 \pm 0.04$ \\
\hline & G4 & $0.05 \pm 0.05$ & $0.07 \pm 0.05^{*}$ & $0.07 \pm 0.04^{*}$ & $0.05 \pm 0.04$ \\
\hline \multirow[t]{4}{*}{ 4. Sodium urinary excretion - $\mathrm{EU}_{\mathrm{Na}^{+}}\left(\mu \mathrm{Eq} \cdot \mathrm{min}^{-1}\right)$} & G1 & $67 \pm 39$ & $47 \pm 29$ & $47 \pm 37$ & $39 \pm 61$ \\
\hline & $\mathrm{G} 2$ & $34 \pm 22$ & $42 \pm 34$ & $36 \pm 21$ & $38 \pm 29$ \\
\hline & G3 & $72 \pm 91$ & $155 \pm 106^{*}$ & $126 \pm 82^{*}$ & $88 \pm 72$ \\
\hline & G4 & $51 \pm 40$ & $111 \pm 93^{*}$ & $134 \pm 81^{*}$ & $111 \pm 94^{*}$ \\
\hline \multirow[t]{4}{*}{ 5. Effective renal plasma flow - ERPF $\left(\mathrm{ml} \cdot \mathrm{kg}^{-1} \cdot \mathrm{min}^{-1}\right)$} & G1 & $18.9 \pm 26$ & $17.3 \pm 12$ & $10.6 \pm 6$ & $11.3 \pm 6$ \\
\hline & G2 & $10.2 \pm 10$ & $9.2 \pm 5$ & $8.7 \pm 6$ & $8.9 \pm 5$ \\
\hline & G3 & $13.4 \pm 8$ & $12.8 \pm 9$ & $9.7 \pm 5$ & $10.0 \pm 4$ \\
\hline & G4 & $13.0 \pm 8$ & $11.8 \pm 6$ & $13.9 \pm 7$ & $17.1 \pm 8^{*}$ \\
\hline
\end{tabular}

${ }^{*} p<0.05$

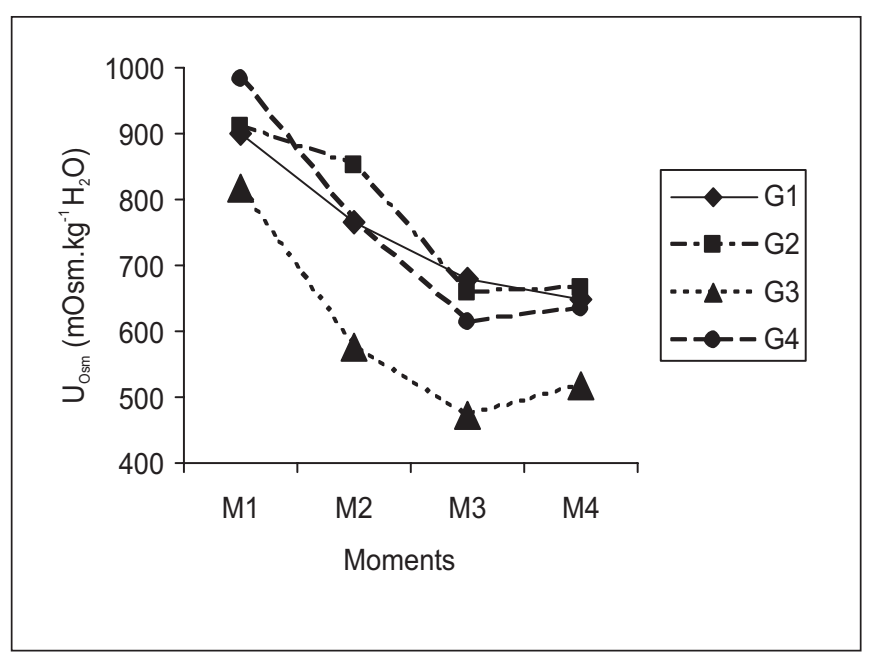

Figure 1 - Urinary Osmolality $\mathrm{U}_{\mathrm{Osm}}\left(\mathrm{mOsm} \cdot \mathrm{kg}^{-1} \mathrm{H}_{2} \mathrm{O}\right)$ in Relation to Time (minutes)

G1 (control group); G2 (dopamine group); G3 (SH group); G4 ( $\mathrm{SH}+$ dopamine group)
$\mathrm{SH}$ infusion associated to dopamine has increased: 1) heart rate $(F=10.52 p<0.05) ; 2)$ ERPF (Table I and Figure 5) group effect in each moment M4 F = 3.06 $p<0.05(\mathrm{G} 1=\mathrm{G} 2=\mathrm{G} 3)<$ G4 and moment effect in each group G4: $F=5.64 p<0.05$ (M1 $=\mathrm{M} 2=\mathrm{M} 3)<\mathrm{M} 4 ; 3)$ Plasma sodium $(F=6.32 \mathrm{p}<0.05)$; 4) Sodium urinary excretion (Table I and Figure 4 ) moment effect in each group $\mathrm{G} 4: \mathrm{F}=7.77 \mathrm{p}<0.05 \mathrm{M} 1<(\mathrm{M} 2=\mathrm{M} 3=\mathrm{M} 4)$. There has been also decrease in: 1) systemic vascular resistance index $(F=13.87 p<0.05) ; 2)$ plasma potassium $(F=10.9 p<$ 0.05).

\section{DISCUSSION}

Continuous infusion of sodium thiopental associated to fentanyl has produced hemodynamic and renal stability and, as a consequence, there have been no changes in major renal and cardiovascular parameters. High urinary osmolality values have decreased from moment M1 to M4. Similarly to other studies, these high osmolality values have shown that water restriction simulating preoperative fast was enough to contract extracellular volume.

It is known that dopamine has a dose-dependent action based on its activity on dopaminergic, $\beta$-adrenergic or Vol. 53, № 5, Setembro - Outubro, 2003 


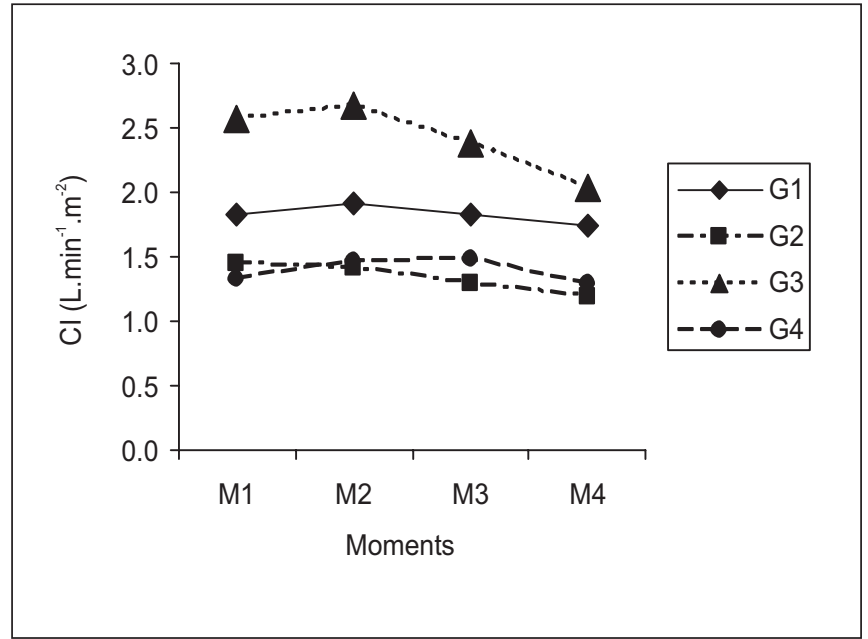

Figure 2 - Cardiac Index Cl (L. $\left.\mathrm{min}^{-1} \cdot \mathrm{m}^{-2}\right)$ in Relation to Time (minutes)

G1 (control group); G2 (dopamine group); G3 (SH group); G4 ( $\mathrm{SH}+$ dopamine group)

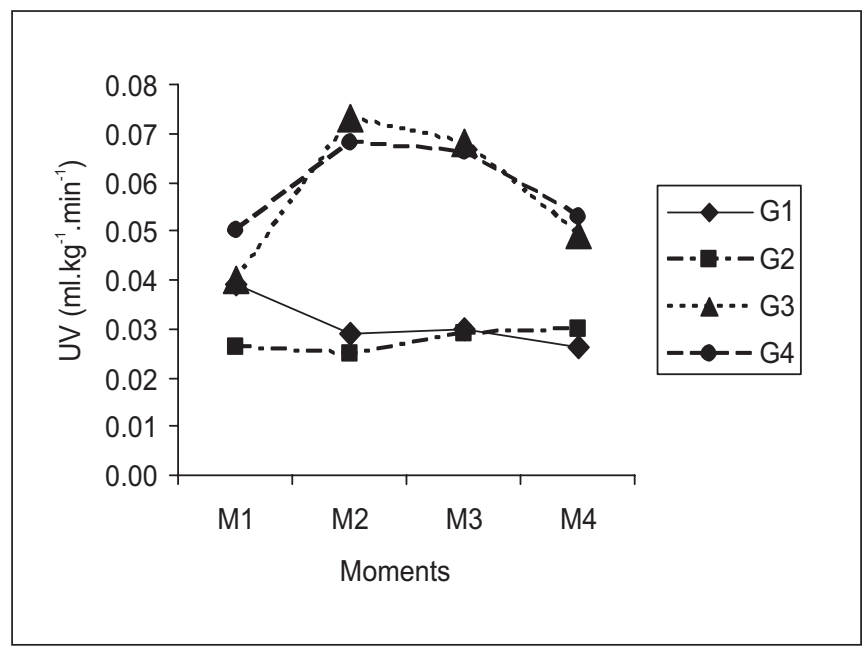

Figure 3 - Urinary Volume - UV $\left(\mathrm{ml}^{\mathrm{kg}} \mathrm{kg}^{-1} \cdot \mathrm{min}^{-1}\right)$ in Relation to Time (minutes)

G1 (control group); G2 (dopamine group); G3 (SH group); G4 (SH + dopamine group)

$\alpha$-adrenergic receptors. Dopamine infusion above 2.5 $\mu \mathrm{g} . \mathrm{kg}^{-1} \cdot \mathrm{min}^{-1}$ has cardiovascular effects through $\beta$-adrenergic receptors ${ }^{11,12}$. Lower doses in general have minor systemic effects, but some studies suggest significant individual variations. In normal individuals, $2.5 \mu \mathrm{g} \cdot \mathrm{kg}^{-1} \cdot \mathrm{min}^{-1}$ dopamine has decreased systemic vascular resistance in $27 \%{ }^{11}$.

In our study, heart rate (HR), cardiac index $(\mathrm{Cl})$, inferior vena cava pressure (IVCP) and peripheral vascular resistance index (PVRI) were not changed, similarly to what has been shown by most studies using $2 \mu \mathrm{g} \cdot \mathrm{kg}^{-1} \cdot \mathrm{min}^{-1}$ dopamine. MBP has decreased after dopamine infusion, showing that in such concentrations, this catecholamine has vasodilating effects.

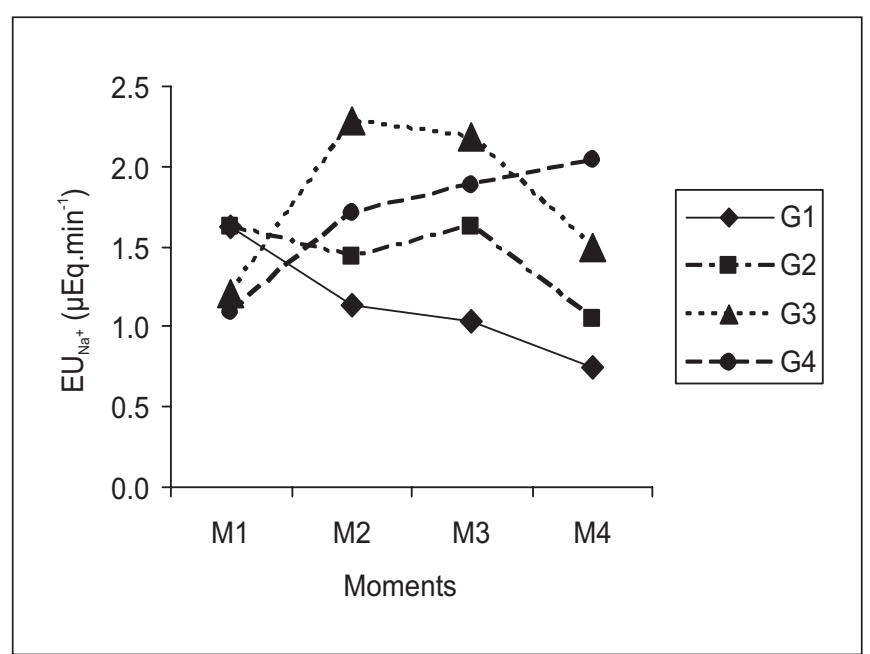

Figure 4 - Sodium Urinary Excretion $\mathrm{EU}_{\mathrm{Na}^{+}}\left(\mu \mathrm{Eq} \cdot \mathrm{min}^{-1}\right)$ in Relation to Time (minutes)

G1 (control group); G2 (dopamine group); G3 (SH group); G4 ( $\mathrm{SH}+$ dopamine group)

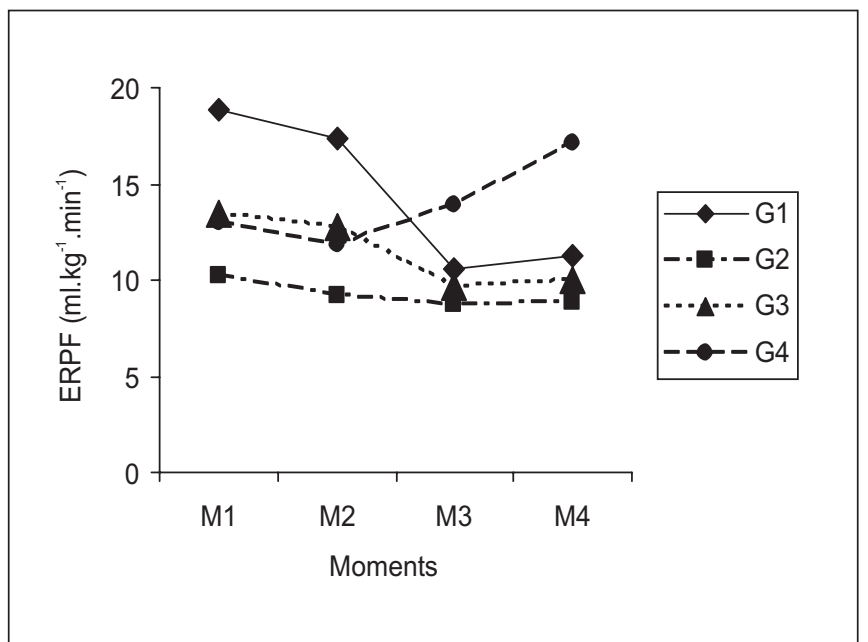

Figure 5 - Effective Renal Plasma Flow - ERPF $\left(\mathrm{ml}^{\mathrm{kg}} \mathrm{kg}^{-1} \cdot \mathrm{min}^{-1}\right)$ in Relation to Time (minutes)

G1 (control group); G2 (dopamine group); G3 (SH group); G4 ( $\mathrm{SH}+$ dopamine group)

This effect, however, was not intense, maintaining such attribute within normal limits.

After some studies ${ }^{13-15}$ have shown that dopamine increases RBF and urinary output, low dopamine doses started to be the option to prevent renal problems in critical patients. The increase in urinary volume during acute oliguric tubular necrosis has been shown with dopamine infusion ${ }^{2}$. Urinary volume and creatinine clearance increase with dopamine in patients submitted to liver transplantation ${ }^{4}$. These results were not further confirmed ${ }^{5}$, when authors have not found increased diuresis with dopamine. It is suggested that patients' hydration status is the possible cause for this discrepancy among different studies. 
There are studies with different patients (healthy, critically ill, with intracranial hypertension, in the postoperative period of obstructive jaundice and cardiac surgery), showing increased urinary volume with dopamine infusion ${ }^{1,14,16}$; however, differently from the animals of our experiment, these were euvolemic individuals.

Our results are also in line with another study ${ }^{13}$ using 0.5 $\mu \mathrm{g} \cdot \mathrm{kg}^{-1} \cdot \mathrm{min}^{-1}$ dopamine infusion, which has not found urinary output, free water clearance and plasma osmolality changes, but has shown renal vascular resistance decrease. Urinary volume was also not increased with dopamine infusion in premature neonates ${ }^{17}$. Authors believe that this could be due to volume retraction, which was evidenced by high urinary osmolality values. Dopamine administration in the preoperative period of cardiac surgeries has caused diuresis and natriuresis increase solely in previously hydrated patients, while in fast patients with water restriction there have been no such changes ${ }^{6}$. Oliguric patients under intensive care had no significant urinary excretion and sodium fractional excretion increase ${ }^{16}$.

Based on the results of those studies and of our experiment, one may suggest that patients need normal or increased extracellular volume to benefit from dopamine diuretic and natriuretic effects.

In our study, dopamine infusion has increased G4 (dopamine associated to $\mathrm{SH}$ ) ERPF, showing renal dopamine vasodilating effect, confirmed by decreased RVR. Dopamine, however, may also produce concomitant splancnic vasodilatation. This splancnic vasodilating effect may further decrease circulating volume ${ }^{18}$.

There is a study showing hematocrit increase with high dopamine doses, but not with low doses ${ }^{19}$. Hematocrit increase in our study may be attributed to water restriction during the experiment.

The infusion of $7.5 \%$ hypertonic sodium chloride solution increases cardiac output, MBP and IVCP, but decreases total peripheral vascular resistance ${ }^{7,10,20}$. Although this total peripheral vascular resistance decrease is partially the result of intravascular volume expansion, hyperosmolality direct vasodilating action may also contribute ${ }^{10}$. Hyperosmolality direct vasodilating effect may help to explain cardiac index increase in our study. Hypertonic sodium chloride solution rapidly restores intravascular volume by uptaking water from the intracellular space to the interstitial and intravascular space ${ }^{21}$. Cardiac output improvement seems to be independent of preload improvement. Cardiac output increase may be caused by a direct effect on contractility ${ }^{22}$ or by extensive pre-capillary vasodilatation ${ }^{10,23}$.

Hypertonic sodium chloride solution infusion has not changed MBP and IVCP, differently from other studies showing increase in those parameters ${ }^{7,10}$. In our study, heart rate has also not changed in G3 and G4. The possible explanation for such results might be water restriction imposed to dogs and anesthetic stability achieved by continuous sodium thiopental and fentanyl infusion.
As expected, cardiac index has increased in G3, similarly to other studies ${ }^{8-10,20,24,25}$ which have shown cardiac output increase with $\mathrm{SH}$ infusion, but this was a transient increase ${ }^{10,20}$.

In our study, decreased PVRI in G3 and G4 after hypertonic sodium chloride solution infusion is in line with several authors' findings ${ }^{10,20,25}$

In swine, hypertonic sodium chloride solution infusion increases cardiac output and RSF ${ }^{24}$.

Our study is in line with other studies ${ }^{7,10,20,24,26}$, which have also found urinary volume, plasma potassium, sodium urinary excretion and plasma osmolality increase with hypertonic solution infusion. SH promotes extracellular volume expansion and, as it has been already said, this leads to RAA (Rennin-Angiotensin-Aldosterone) system inhibition, thus increasing sodium urinary excretion. This explains the statistically significant increase in sodium urinary excretion in G3 and G4 in our experiment. It also explains the increase in sodium fractional excretion in these groups, being significant in G3.

Plasma potassium decrease in groups receiving hypertonic sodium chloride solution was in line with several studies $10,20,25$. It is suggested that there might have been hyponatremia by the passage of $\mathrm{K}^{+}$to the intracellular space, although resolution has been spontaneous.

In conclusion: (a) $2 \mu \mathrm{g} \cdot \mathrm{kg}^{-1} \cdot \mathrm{min}^{-1}$ dopamine has significantly decreased mean blood pressure and renal vascular resistance; (b) hypertonic sodium chloride solution has significantly changed cardiovascular hemodynamics with inotropic effects, has changed renal hemodynamics with increased sodium and potassium clearance. It has also changed renal function with increased urinary volume, sodium and potassium urinary excretion and sodium fractional excretion. It has decreased urinary osmolality. All changes were transient; (c) hypertonic sodium chloride solution associated to dopamine has significantly changed cardiovascular hemodynamics with chronotropic and inotropic effects. It has also changed renal hemodynamics with increased effective renal plasma flow and free water clearance. It has changed renal function with increased sodium and potassium urinary excretion and decreased osmolality.

\section{REFERÊNCIAS - REFERENCES}

01. Fontana F, Bernardi P, Ruffini A et al - Atrial natriuretic factor after dopamine infusion in healthy subjects and in congestive heart failure. Eur Heart J, 1991;12:803-806.

02. Henderson IS, Beattie TJ, Kennedy AC - Dopamine hydrochloride in oliguric states. Lancet, 1980;18: 827-829.

03. Smit AJ, Meijer S, Wesseling $\mathrm{H}$ et al - Impaired renal hemodynamic but conserved natriuretic response to dopamine in patients with renal disease. Nephron, 1989;52:338-346.

04. Polson RJ, Park GR, Lindop MJ et al - The prevention of renal impairment in patients undergoing orthotopic liver grafting by infusion of low dose dopamine. Anaesthesia, 1987;42:15-19.

05. Swygert TH, Roberts C, Valek TR et al - Effects of intraoperative low-dose dopamine on renal function in liver transplant recipients. Anesthesiology, 1991;75:571-576. 
06. Bryan AG, Bolsin SN, Vianna PTG et al - Modification of the diuretic and natriuretic effects of a dopamine infusion by fluid loading in preoperative cardiac surgical patients. J Cardiothorac Vasc Anesth, 1995;9:158-163.

07. Felippe Jr JDE, Timoner J, Velasco IT et al - Treatment of refractory hypovolaemic shock by $7.5 \%$ sodium chloride injections. Lancet, 1980;8:1002-1004.

08. Velasco IT, Pontieri V, Rocha e Silva JRM et al - Hyperosmotic $\mathrm{NaCl}$ and severe hemorrhagic shock. Am J Physiol, 1980;239: H664-673.

09. Lopes OU, Pontieri V, Rocha e Silva JRM et al - Hyperosmotic $\mathrm{NaCl}$ and severe hemorrhagic shock: role of the innervated lung. Am J Phisiol, 1981;241:H883-890.

10. Nakayama SI, Sibley L, Gunther RA et al. Small-volume resuscitation with hypertonic saline $\left(2400 \mathrm{mOsm} . \mathrm{I}^{-1}\right)$ during hemorrhagic shock. Circ Shock, 1984;13:149-159.

11. Ruttimann $Y$, Chioléro $R$, Jéquier $E$ et al - Effects of dopamine on total oxygen consumption and oxygen delivery in healthy men. Am J Physiol, 1989;257:E541-546.

12. Szerlip HM - Renal-dose dopamine: fact and fiction. Ann Intern Med, 1991;115:153-154.

13. Schwartz LB, Bissell MG, Murphy M et al. - Renal effects of dopamine in vascular surgical patients. J Vasc Surg, 1988;8: 367-374.

14. Schoors DF, Dupont AG - Further studies on the mechanism of the natriuretic response to low-dose dopamine in man: effect on lithium clearance and nephrogenic cAMP formation. Eur J Clin Invest, 1990;20:385-391.

15. Graves TA, Cioffi WG, Vaughan GM et al - The renal effects of low-dose dopamine in thermally injured patients. J Trauma, 1993;35:97-103

16. Flancbaum L, Choban PS, Dasta JF - Quantitative effects of low-dose dopamine on urine output in oliguric surgical intensive care unit patients. Crit Care Med, 1994;22:61-66.

17. Cuevas L, Yeh TF, John EG et al - The effects of low-dose dopamine infusion on cardiopulmonary and renal status in premature newborns with respiratory distress syndrome. Am J Dis Child, 1991;145:799-803.

18. Durkin RJ, Winter SM - Reversal of hepatorenal syndrome with the combination of norepinephrine and dopamine. Crit Care Med, 1995;23:202-204.

19. Ball SG, Tree M, Morton JJ et al - Circulating dopamine: its effect on the plasma concentrations of catecholamines, renin, angiotensin, aldosterone and vasopressin in the conscious dog. Clin Sci, 1981;61:417-422.

20. Armistead Jr ChW, Vicent JL, Preiser JCh et al - Hypertonic saline solution-hetastarch for fluid resuscitation in experimental septic shock. Anesth Analg, 1989;69:714-720.

21. Moss GS, Gould SA - Hypertonic saline. Prog Clin Biol Res, 1989;299:293-302.

22. Rowe GG, Mc Kenna DH, Corliss RJ et al - Hemodynamic effects of hypertonic sodium chloride. J Appl Physiol, 1972;32: 182-184.

23. Gazitúa S, Scott JB, Swindall B et al - Resistance responses to local changes in plasma osmolality in three vascular beds. Am J Phisiol, 1971;220:384-391.

24. Sondeen JL, Gonzaludo GA, Hoveday JA et al - Hypertonic saline/dextran improves renal function after hemorrhage in conscious swine. Resuscitation, 1990;20:231-241.
25. Tobias TA, Schertel ER, Schamall LM et al - Comparative effects of $7.5 \% \mathrm{NaCl}$ in $6 \%$ Dextran 70 and $0.9 \% \mathrm{NaCl}$ on cardiorespiratory parameters after cardiac output controlled resuscitation from canine hemorrhagic shock. Circ Shock, 1993;398:139-146

26. Matteucci MJ, Wisner DH, Gunther RA et al - Effects of hypertonic and isotonic fluid infusion on the flash evoked potential in rats: hemorrhage, resuscitation and hypernatremia. $\mathrm{J}$ Trauma, 1993;34:1-7.

\section{RESUMEN}

Verderese MAL, Vianna PTG - Efectos Renales y Cardiovasculares de la Infusión de Dopamina y de la Solución de Clorato de Sodio $7,5 \%$. Estudio experimental en Canes con Restricción Hídrica

JUSTIFICATIVA Y OBJETIVOS: Es controvertido el uso de la infusión de dopamina en la protección renal. El objetivo de esta pesquisa fue estudiar el efecto de la dopamina, de la solución hipertónica y de la asociación de ambas en canes con restricción hídrica, simulando el ayuno pre-operatorio.

MÉTODO: Fueron estudiados, en 32 canes anestesiados con tiopental sódico y fentanil, los siguientes parámetros de la función renal: flujo plasmático efectivo renal (depuración de para-aminohipurato de sodio), ritmo de filtración glomerular (depuración de creatinina) y las depuraciones de sodio, de potasio y osmolar, excreción fraccionaria de sodio y potasio, excreción de sodio y potasio y la resistencia vascular renal. Los parámetros cardiovasculares fueron: presión arterial media, frecuencia cardíaca, presión de la vena cava inferior, índice cardíaco, hematócrito e índice de resistencia vascular periférica. Los animales fueron subdivididos, a través de sorteo, en 4 grupos experimentales: Grupo 1 - $G 1$ ( $n=8)$ - grupo control; Grupo 2 - G2 ( $n=8)$ infusión de dopamina (2 $\left.\mu g \cdot \mathrm{kg}^{-1} \cdot \mathrm{min}^{-1}\right)$, Grupo 3 - $\mathrm{G} 3(n=8)$ solución de clorato de sodio a $7,5 \%\left(2 \mathrm{ml} . \mathrm{kg}^{-1}\right)$ y Grupo 4 - G4 $(n=8)$ - asociación de dopamina $\left(2 \mu \mathrm{g} \cdot \mathrm{kg}^{-1} \cdot \mathrm{min}^{-1}\right)$ y clorato de sodio a $7,5 \%\left(2 \mathrm{ml} \cdot \mathrm{kg}^{-1}\right)$. Los grupos tuvieron cuatro partes experimentales y cada momento con duración de 30 minutos, comprendiendo los momentos M1, M2, M3 y M4.

RESULTADOS: El grupo de la dopamina (G2) presentó diminución de la presión arterial media, de la resistencia vascular renal y de la excreción de potasio. El grupo de la solución hipertónica de clorato de sodio (G3) presentó aumento del índice cardíaco, del volumen urinario, de la depuración de sodio y de potasio, de la excreción urinaria de sodio y potasio y de la excreción fraccionaria de sodio. En el grupo de la solución hipertónica de clorato de sodio asociada a la dopamina (G4), ocurrió elevación de la frecuencia cardíaca, del índice cardíaco, del flujo plasmático efectivo renal y de la excreción urinaria de sodio; ocurrió también diminución del índice de resistencia vascular sistémica y del potasio plasmático.

CONCLUSIONES: De este estudio se concluye que la solución hipertónica de clorato de sodio fue capaz de mejorar las condiciones hemodinámicas y, consecuentemente, la función renal de canes bajo restricción hídrica de 12 horas. Lo mismo no aconteció con la infusión de $2 \mu \mathrm{g} \cdot \mathrm{kg}^{-1} \cdot \mathrm{min}^{-1}$ de dopamina que, en situación similar, no causó aumento de la diuresis y de la excreción de sodio. 